\title{
Motion by Stopping: Rectifying Brownian Motion of Non-spherical Particles
}

\author{
Susan Sporer, ${ }^{1}$ Christian Goll, ${ }^{1}$ and Klaus Mecke ${ }^{1}$ \\ ${ }^{1}$ Institut für Theoretische Physik, Universität Erlangen-Nürnberg, \\ Staudtstraße 7, 91058 Erlangen, Germany
}

(Dated: October 23, 2018)

\begin{abstract}
We show that Brownian motion is spatially not symmetric for mesoscopic particles embedded in a fluid if the particle is not in thermal equilibrium and its shape is not spherical. In view of applications on molecular motors in biological cells, we sustain non-equilibrium by stopping a non-spherical particle at periodic sites along a filament. Molecular dynamics simulations in a Lennard-Jones fluid demonstrate that directed motion is possible without a ratchet potential or temperature gradients if the asymmetric non-equilibrium relaxation process is hindered by external stopping. Analytic calculations in the ideal gas limit show that motion even against a fluid drift is possible and that the direction of motion can be controlled by the shape of the particle, which is completely characterized by tensorial Minkowski functionals.
\end{abstract}

PACS numbers: 05.70.Ln, 05.40.Jc, 87.15.Vv, 87.16.Ac 
Mesoscopic particles dissolved in a fluid are expected to perform symmetric thermal fluctuations around a mean position [1, 2]. The shape of the particles is irrelevant for this so-called Brownian motion - as long as the particle is in thermal equilibrium with the fluid. Consequently, a net transport in a preferred direction is not possible without applying an external force which breaks the spatial symmetry. In the past, several models for Brownian motors have been proposed based on different methods of rectifying thermal noise; for instance, by an asymmetric external potential which is switched on and off periodically (for a review see Ref. [3]). Another concept is based on two heatbaths at different temperatures such as the Feynman ratchet [4] and its simplification by Van den Broeck [5]. It was shown that directed Brownian motion can be achieved as long as the two reservoirs have different temperatures and spatial symmetry is broken in some way. This letter demonstrates that a Brownian motor can be built even in a single heatbath without violating the second law of thermodynamics. Hence, it is a possible theoretical model for molecular motors in biological cells. We show that a steady state motion of non-spherical particles is obtained if their relaxation towards equilibrium is prohibited by periodical stopping.

To illustrate the concept we restrict the system to two dimensions and follow Ref. [5]. Since the spatial symmetry breaking is a prerequisite for net transport, we consider an asymmetric motor $K$ of mass $M$ in a single heatbath at temperature $\mathrm{T}$. The motion of the motor is restricted to a one-dimensional track, here the $x$-axis, so that the motor velocity can be written as $\widetilde{\mathbf{V}}=\left(V \sqrt{k_{B} T / M}, 0\right)$ with the normalized velocity $V$ in $x$-direction. Furthermore, elastic interactions are assumed between the motor $K$ and the surrounding fluid particles. In contrast to Refs. [7, 8] there is no dissipation involved in this collision. However, due to the confinement only the axial momentum component is conserved. In thermal equilibrium, directed motion in a single heatbath is prohibited by the second law of thermodynamics. The motor $K$ behaves like a Brownian particle with a Maxwellian velocity distribution with mean $\langle V\rangle=0$ and variance $\left\langle V^{2}\right\rangle=1$; independent of its particular shape. Hence, in order to get directed motion, we have to sustain a non-equilibrium state.

Our model is motivated by a molecular motor in a cell, e.g. kinesin, that binds to a filament after every step [6]. Similarly, our particle is stopped at periodically spaced binding sites along the track, i.e. its kinetic energy is set to zero whenever it reaches a binding site. Thermodynamically, of course, the stopping requires a greater amount of work than the decrease in entropy; here $\frac{1}{2} k_{B} T$ from the reduction of the Maxwellian distribution for $V$ to the velocity distribution $P(V)=\delta(V)$ for the stopped particle. After binding the motor is released again and starts to relax towards 
thermal equilibrium. By means of collisions between fluid particles and the motor, kinetic energy is transfered from the fluid to the motor until the motor has the same effective temperature as the surrounding bath.

In the following, we show that this non-equilibrium relaxation process is asymmetric due to the asymmetry of the particle. This asymmetric relaxation process can be used to rectify thermal fluctuations. To test this hypothesis, we performed 2D molecular dynamics simulations of an asymmetric particle in a two-dimensional fluid. The interaction between the fluid particles is described by the Lennard-Jones $(\mathrm{LJ})$ potential $\mathcal{U}(r)=4 \mathcal{E}\left(\left(\frac{\sigma}{r}\right)^{12}-\left(\frac{\sigma}{r}\right)^{6}\right)$ where $r$ is the distance between two particles with diameter $\sigma$ and $\mathcal{E}$ is the depth of the potential well. In the following, all lengths are given in units of $\sigma$, energies in units of $\mathcal{E}$ and times are expressed in units of $t_{0}=$ $\sigma \sqrt{m / \mathcal{E}}$ where $m$ denotes the mass of the fluid particles. The simulation box with edge length $100 \sigma$ contains $n=800$ fluid particles yielding a particle density $\varrho \approx 0.08 \sigma^{-2}$. The temperature is set to $T=3 \mathcal{E} / k_{B}$ and the overall simulation time was $2000 t_{0}$. With these parameters the LJ fluid is in the gas phase without any long range correlations. Periodic boundary conditions are used and also a cut-off radius of $2.5 \sigma$ in $\mathcal{U}(r)$ to limit the range of the potential.

As illustrated in Fig. 1 the motor is built of $N$ stiffly-linked fluid particles which interact with the surrounding fluid also by a Lennard-Jones potential. Consequently, the motor mass $M=N m$ scales with the mass $m$ of the fluid particles. The motion of the motor is confined to a one-

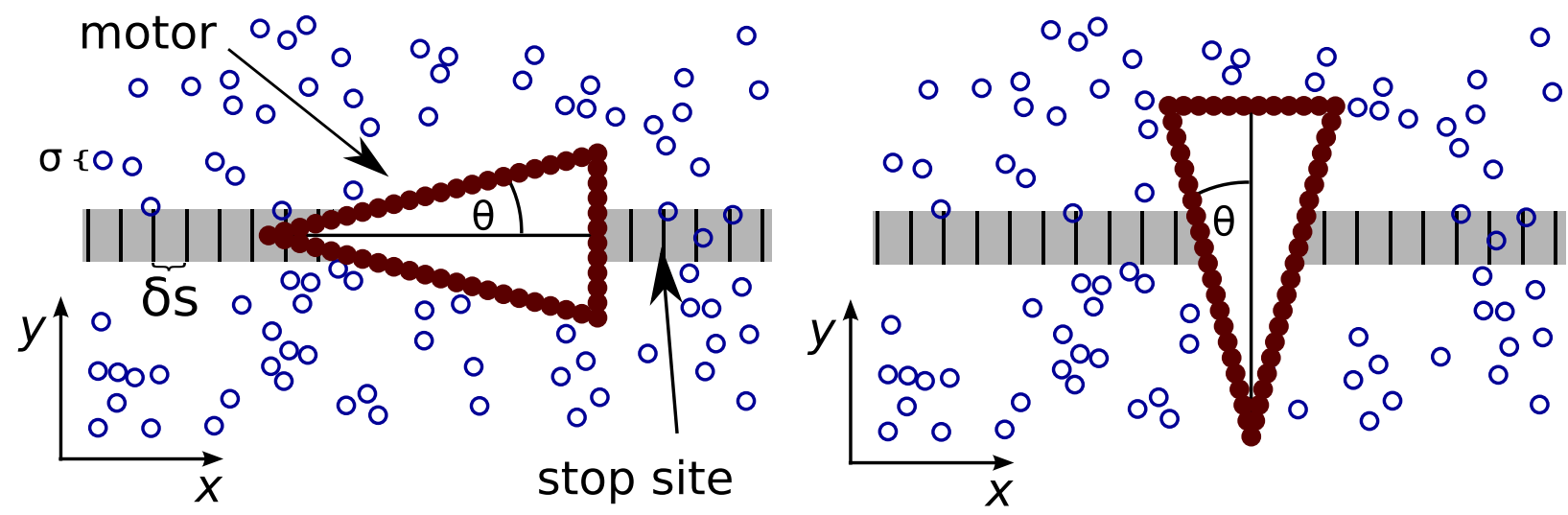

FIG. 1: An asymmetrically shaped motor (here a triangle) is built from fluid particles and placed in a Lennard-Jones fluid. Its motion is restricted to a one-dimensional track with periodically spaced binding sites along the $\mathrm{x}$-axis. If the motor's center of mass crosses a binding site, the velocity of the motor is set to zero. To test the importance of the motor's shape for the momentum transfer during collisions with fluid particles we orient the triangle in two different ways. 


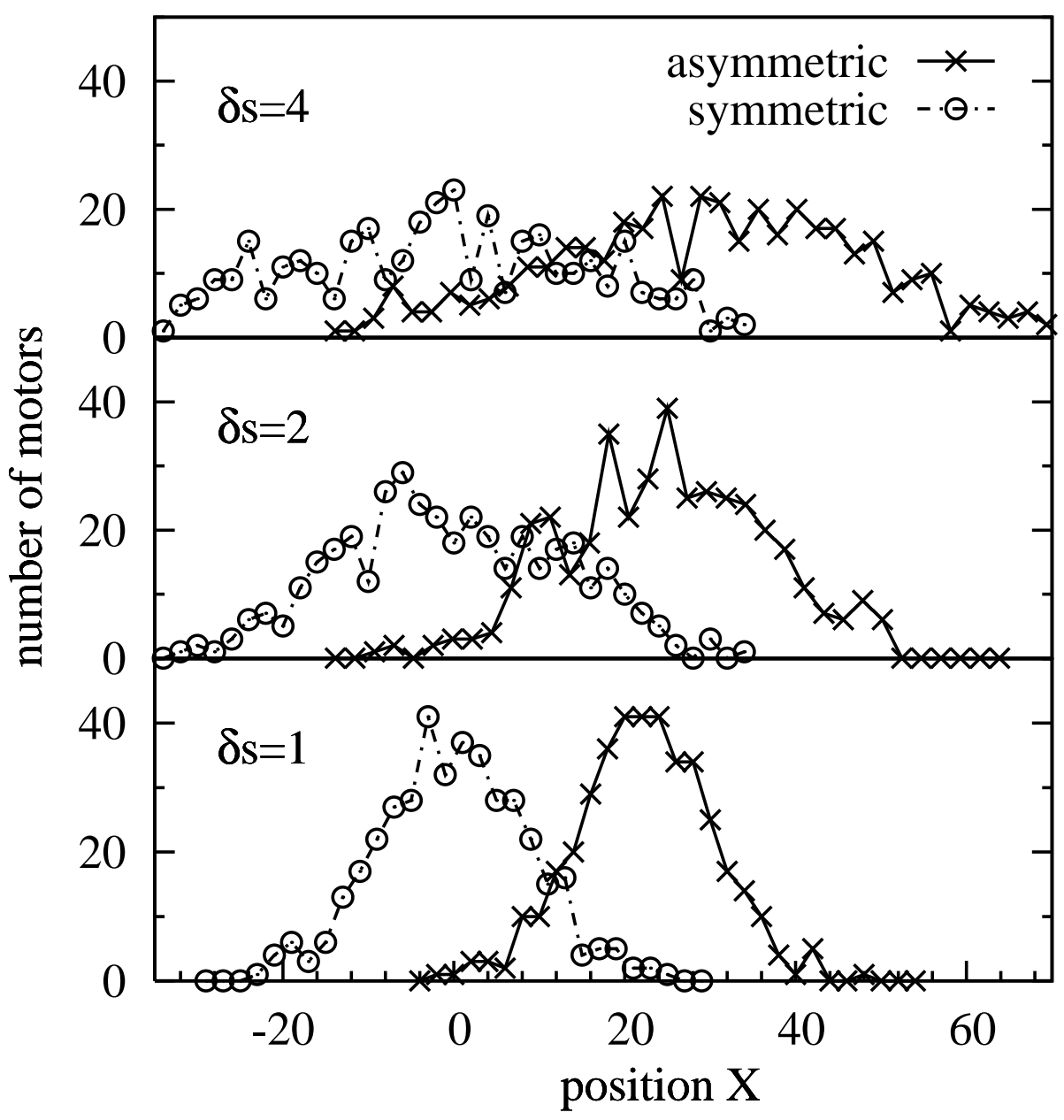

FIG. 2: Histograms of end positions at time $t=2000 t_{0}$ of a triangular motor where the distance between stopping sites is $\delta s$ (see Fig. 1). The asymmetrically oriented motor moves preferable to positive $\mathrm{X}$-values whereas the symmetric triangle diffuses around $X=0$. A discrete spacing is visible since the motors accumulate around the stopping sites. 400 realisations are used with integration step $\delta t=0.001 t_{0}$ and 2000000 steps. With decreasing distances $\delta s$ between stopping sites the distribution becomes narrower, as expected for a hindered diffusion process.

dimensional track with periodically spaced stopping sites. Every time the motor's center of mass reaches one of those sites, the velocity of the motor is set to zero and the corresponding kinetic energy is re-transferred to the fluid.

Consider a motor with the shape of an isosceles triangle of height $10 \sigma$ and basis length $20 \sigma$ which corresponds to an apex angle of $\theta=0.25268, N=53$ and mass $M=53 \mathrm{~m}$. The motor is 
placed in the beginning $(t=0)$ at the position $X=0$. Figs. 2 and 3 show the simulation results for the motion of the motor. Note, that for the chosen parameters the typical time between two successive stops of the motor is smaller than the relaxation time into equilibrium. In case of an isosceles triangle, the motor can be oriented symmetrically or asymmetrically with respect to its direction of motion (Fig.11), to test the importance of the shape. Fig.2 presents the distribution over multiple runs of the end positions, i.e. the positions of the motors after time $2000 t_{0}$, for different stopping distances $\delta s$. If the triangle is placed with the apex onto the track, i.e. asymmetric with respect to the direction of motion, the maximum of the distribution of the end positions is shifted to positive values $x>0$. Hence, on average, the triangle travels a certain distance towards its basis. This is in accordance with the results shown in Fig. 3 where a net speed of about $\langle v\rangle=0.011 \frac{\sigma}{t_{0}}$ in the direction of the triangle basis can be observed for asymmetric orientation. In contrast, aligning the triangle basis parallel to the $x$-axis conserves spatial symmetry, i.e. mirror-symmetry with respect to the $y$-axis, and therefore causes the mean position to fluctuate around zero without any net motion (see Fig. 3). The end positions of the motors are in this case symmetrically distributed around the origin (see Fig. 2). This shows two things: first, the proposed model demonstrates the feasibility of rectifying Brownian motion in a single heatbath by stopping the particle at periodic sites. Second, it is sufficient to break the spatial symmetry by the shape of the particle, so that an asymmetric external potential is not necessary.

To get a better insight into the microscopic mechanism of the driving force, we calculate analytically the stochastic dynamics of this non-equilibrium relaxation process for a simplified model. The interaction between the fluid and the motor is no longer a Lennard-Jones potential but an ideal hard sphere interaction with infinitesimal interaction time. The total energy is conserved as well as the momentum in $x$-direction and tangential to the particle's surface. Details of this model can be found in Ref. [5].

Furthermore, we assume an infinite heatbath, so that the temperature is constant and the velocity distribution of the fluid is a Maxwell distribution $\Phi_{\boldsymbol{u}}(\boldsymbol{v})=\frac{m}{2 \pi k_{B} T} \exp \left(-\frac{m}{2 k_{B} T}(\boldsymbol{v}-\boldsymbol{u})^{2}\right)$. To study motion against a drift we introduce an average drift velocity $\boldsymbol{u}$ of the fluid. In the beginning, we assume $\boldsymbol{u}=0$. In order to exclude multiple collisions, we use the ideal gas limit of the fluid and confine the motor shape to convex objects $K$ so that the stochastic motion is a Markov process. This allows us to describe the time dependence of the velocity distribution $P(V, t)$ of the motor by 


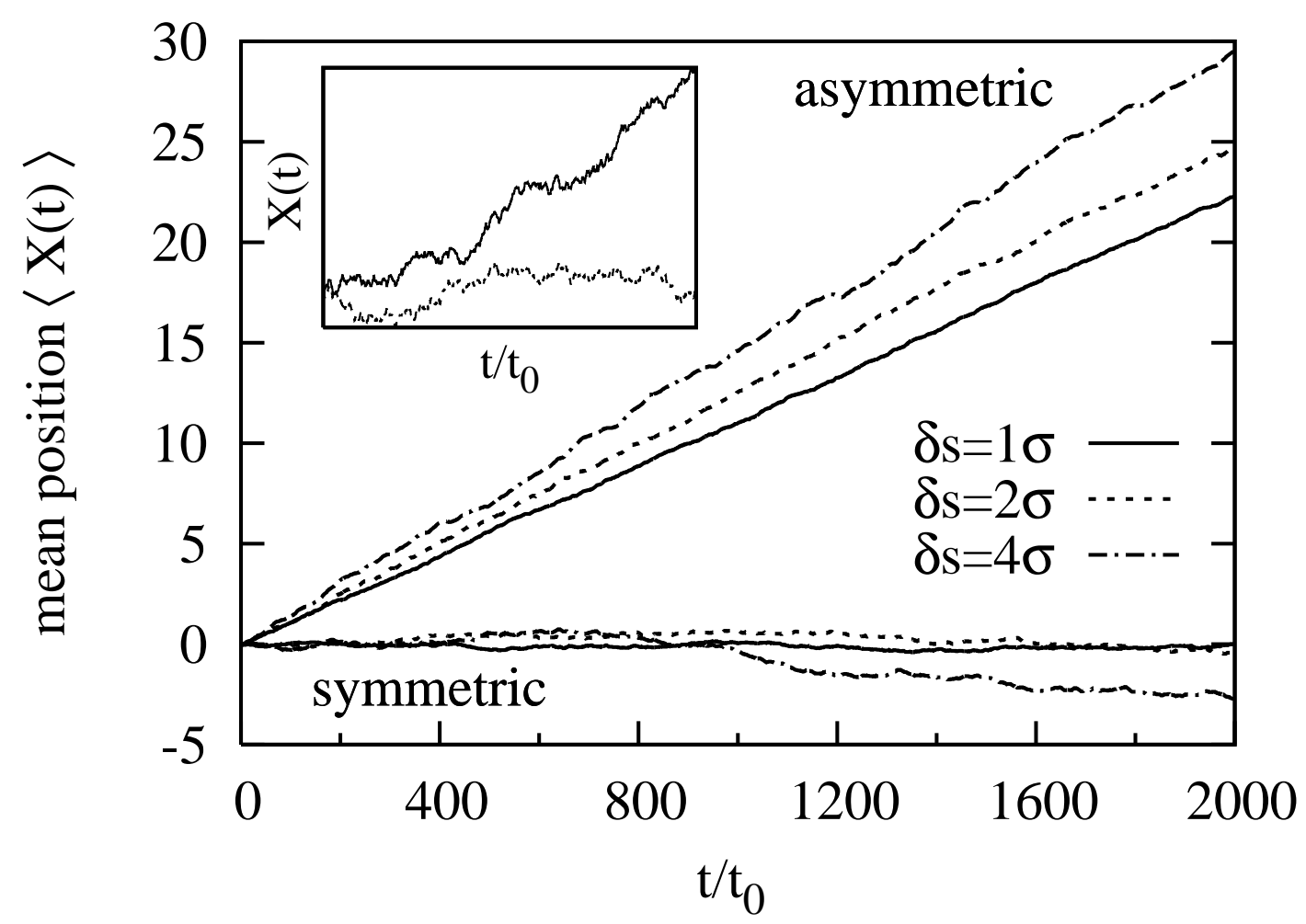

FIG. 3: The average position (400 runs) of an asymmetrically oriented, isosceles triangle of height $10 \sigma$ and basis length $20 \sigma$ moves with a constant velocity in the direction opposite to its sharp edge (i.e., in the direction of its basis). Thus, directed motion is possible when the triangle is oriented asymmetrically with respect to the direction of motion. Otherwise the motor performs symmetric fluctuations around its starting position. Inset: trajectories of individual motors. Note, that the velocity decreases for decreasing distances $\delta s$ between stopping sites if the typical stopping time is smaller than the relaxation time of the motor.

a Master equation with a transition rate [5]

$$
\begin{aligned}
W\left(V^{\prime} \mid V\right) & =\int_{\partial K} d \mathcal{O} \int_{-\infty}^{+\infty} d \boldsymbol{v} \rho \Phi_{0}(\boldsymbol{v})(\widetilde{\mathbf{V}}-\boldsymbol{v}) \cdot \hat{\mathbf{e}}_{\perp} \\
& \times \Theta\left[(\widetilde{\mathbf{V}}-\boldsymbol{v}) \cdot \hat{\mathbf{e}}_{\perp}\right] \delta\left[\widetilde{V}^{\prime}-\widetilde{V}+\frac{\Delta \boldsymbol{p}}{M} \cdot \hat{\mathbf{e}}_{x}\right]
\end{aligned}
$$

$\left(\widetilde{V}=\sqrt{k_{B} T / M} V\right)$ which equals the number of collision processes from $V$ to $V^{\prime}$ per unit time using the dimensionless time $t=8 \epsilon^{2} \sqrt{\frac{k_{B} T \rho}{2 \pi m}} \tilde{t}$. Here, $\hat{\mathbf{e}}_{\|}=(\cos \phi, \sin \phi)$ is the tangential and $\hat{\mathbf{e}}_{\perp}=(\sin \phi,-\cos \phi)$ the normal vector in the collision point $\mathbf{r} \in \partial K$ on the boundary $\partial K$ of the motor and $\phi$ is the polar angle. The momentum transfer of a single collision is then given by $\Delta \boldsymbol{p}=\frac{2 m M \hat{\mathbf{e}}_{\perp}}{M+m\left(\hat{\mathbf{e}}_{\perp} \cdot \hat{\mathbf{e}}_{x}\right)^{2}}(\widetilde{\mathbf{V}}-\boldsymbol{v}) \cdot \hat{\mathbf{e}}_{\perp}$ (see Ref. [5]). Because the Master equation cannot be solved 
exactly, we apply a Kramers-Moyal expansion yielding

$$
\frac{\partial P(V, t)}{\partial t}=\sum_{n=1}^{\infty} \frac{1}{n !}\left(-\frac{\partial}{\partial V}\right)^{n}\left[A_{n}(V, t) P(V, t)\right]
$$

with the coefficients $A_{n}(V, t)=\int d V^{\prime}\left(V^{\prime}-V\right)^{n} W\left(V \mid V^{\prime}\right)$. Different to Ref. [5], we emphasise two important points: (i) the dependence on the shape of the motor can be fully described by tensorial Minkowski functionals $M_{\nu}^{(r, s)}(K)$ of the motor $K$; (ii) the non-stationary solution of Eq. (2) is the essential ingredient to rectify Brownian motion if relaxation is prohibited by stopping sites (for details see Ref. [9]).

Tensorial Minkowski functionals are defined as surface integrals in $d$ dimensions [10]

$$
M_{1}{ }^{(r, s)}(K)=\int_{\partial K} d \mathcal{O} \overbrace{\mathbf{r} \otimes \ldots \otimes \mathbf{r}}^{r-\text { times }} \otimes \overbrace{\hat{\mathbf{e}}_{\perp} \otimes \ldots \otimes \hat{\mathbf{e}}_{\perp}}^{\text {s-times }}
$$

over tensor products of position $\mathbf{r}$ and normal vector $\hat{\mathbf{e}}_{\perp}$ on the surface $\partial K$ of motor $K$. Using $\left(\hat{\mathbf{e}}_{\perp} \cdot \hat{\mathbf{e}}_{x}\right)^{n}=\sin ^{n} \phi$, the $x \ldots x$-components of the first tensorial Minkowski functional in two dimensions can be written as $\left(M_{1}^{(0, n)}(K)\right)_{x \ldots x}^{n-t i m e s}=\int_{\partial K} d \mathcal{O} \sin ^{n} \phi$. For convex bodies $K$ one finds $M_{\nu}^{(0,1)}(K)=0$ so that the first two jump moments read $A_{1}(V)=\tau^{-1}(V+$ $\left.4 V_{\max }\left(V^{2}-1\right)\right)$ and $A_{2}(V)=2 \tau^{-1}\left(1+12 V_{\max } V\right)$. Here, we introduced the relaxation rate $\tau^{-1}=\sqrt{\rho}\left(M_{1}^{(0,2)}(K)\right)_{x x} / 2$ and the maximum velocity

$$
V_{\max }=\frac{\epsilon}{4} \sqrt{\frac{\pi}{8}} \frac{\left(M_{1}^{(0,3)}(K)\right)_{x x x}}{\left(M_{1}^{(0,2)}(K)\right)_{x x}} .
$$

The shape dependence of the stochastic dynamics enters only via these two parameters which can be expressed in terms of Minkowski tensors. These functionals are known in many cases to allow for a complete description of the shape-dependence of physical properties [10]. The other Minkowski tensors in Eq. (3) play a role when the collision rules in Eq. (1) are modified or the drift is non-zero, for instance [9].

From Eq. (2), one immediately obtains the time dependence of the average velocity $\partial_{t}\langle V\rangle=$ $\left\langle A_{1}(V)\right\rangle$ and the average squared velocity $\partial_{t}\left\langle V^{2}\right\rangle=2\left\langle V A_{1}(V)\right\rangle+\left\langle A_{2}(V)\right\rangle$. The equations are coupled to higher moments of $V$ because of the dependence of $A_{n}(V)$ on $V$. However, assuming the mass $m$ of the fluid particles to be small against the motor mass $M$, an expansion in $\epsilon=\sqrt{\frac{m}{M}}$ leads to a decoupling of the differential equations from higher moments when terms of order $\mathcal{O}\left(\epsilon^{4}\right)$ 
or higher are neglected

$$
\begin{aligned}
\frac{\partial\langle V\rangle}{\partial t} & =-\frac{1}{\tau}\left[\langle V\rangle+\left(\left\langle V^{2}\right\rangle-1\right) V_{\max }\right]+\mathcal{O}\left(\epsilon^{4}\right) \\
\frac{\partial\left\langle V^{2}\right\rangle}{\partial t} & =\frac{2}{\tau}\left(1-\left\langle V^{2}\right\rangle\right)+\mathcal{O}\left(\epsilon^{4}\right) .
\end{aligned}
$$

Hence, the solutions give readily the mean velocity $\langle V\rangle(t)=4 V_{\max }\left(e^{-t / \tau}-e^{-2 t / \tau}\right),\left\langle V^{2}\right\rangle(t)=$ $1-e^{-2 t / \tau}$ and the average of the travelled distance $\langle X\rangle(t)=\int_{0}^{t} d t^{\prime} V\left(t^{\prime}\right)=2 V_{\max } \tau\left(1-e^{-t / \tau}\right)^{2}$. The time $\tau$ characterizes the increase of the kinetic energy $\frac{M}{2}\left\langle\widetilde{V}^{2}\right\rangle$ towards its equilibrium value $\frac{1}{2} k_{B} T$, which is the stationary solution of Eq. (5). The relaxation process depends on the shape of the motor only via the maximum of the averaged velocity $V_{\max }$, so that the shape dependence is fully described by the Minkowski functionals of the particle $K$. For instance, the Minkowski functionals of a triangular motor with the top pointing in negative $x$-direction reads $\left(M_{1}^{(0,2)}(K)\right)_{x x}=L(1+\sin \theta)$ and $\left(M_{1}^{(0,3)}(K)\right)_{x x x}=L \cos ^{2} \theta$, so that $V_{\max }=\frac{\epsilon}{4} \sqrt{\frac{\pi}{8}}(1-\sin \theta)$ and $\tau^{-1}=L \sqrt{\rho}(1+\sin \theta) / 2$ depend on the apex angle $\theta$ of the triangle (see Fig. 11).

The shape dependence of this non-equilibrium Brownian motion can be perfectly demonstrated with the 'capped-triangular' motor shown in the inset of Fig. 4(b), whose Minkowski functionals are $\left(M_{1}^{(0,2)}(K)\right)_{x x}=L\left(\frac{\pi}{4}+\sin \theta\right)$ and $\left(M_{1}^{(0,3)}(K)\right)_{x x x}=L\left(\frac{2}{3}-\sin ^{2} \theta\right)$. As shown in Fig. 4(b), the relaxation time $\tau$ decreases with increasing opening angle $\theta$ whereas the maximum average velocity $V_{\max }$ increases with $\theta$ from negative values to positive ones. Interestingly, one can change the direction of motion just by adjusting the shape of the motor. For small $\theta$, i.e. long triangle legs relative to the arc length of the semi-circle, the motor moves towards the semi-circle, whereas it travels towards the triangle if $\theta$ is close to $\frac{\pi}{2}$. Note, that there is exactly one value for $\theta$ for which the motor does not move on average ( $V_{\max }=0$ vanishes) though the motor definitely breaks spatial symmetry.

The relaxation process of the triangular motor is shown in Fig. 4. The average velocity first increases until a maximum value $V_{\max }$ is reached and than decays exponentially towards the equilibrium with vanishing mean velocity. Note, that in the beginning the motor travels an average distance $X_{\max }=2 V_{\max } \tau$ before it fluctuates around the mean position $X_{\max }$ in the long time limit. Shortly after releasing, the motion of the motor has a preferred direction. Thus repeated stopping yields directed motion if it occurs before the system has relaxed completely.

A simple analogy of the rectification mechanism is a piston in a cylinder separating two fluids in thermal equilibrium at the same temperature. If the piston is arrested or has an infinite mass, one finds in thermodynamic textbooks that the averaged momentum transfer on one side per unit 

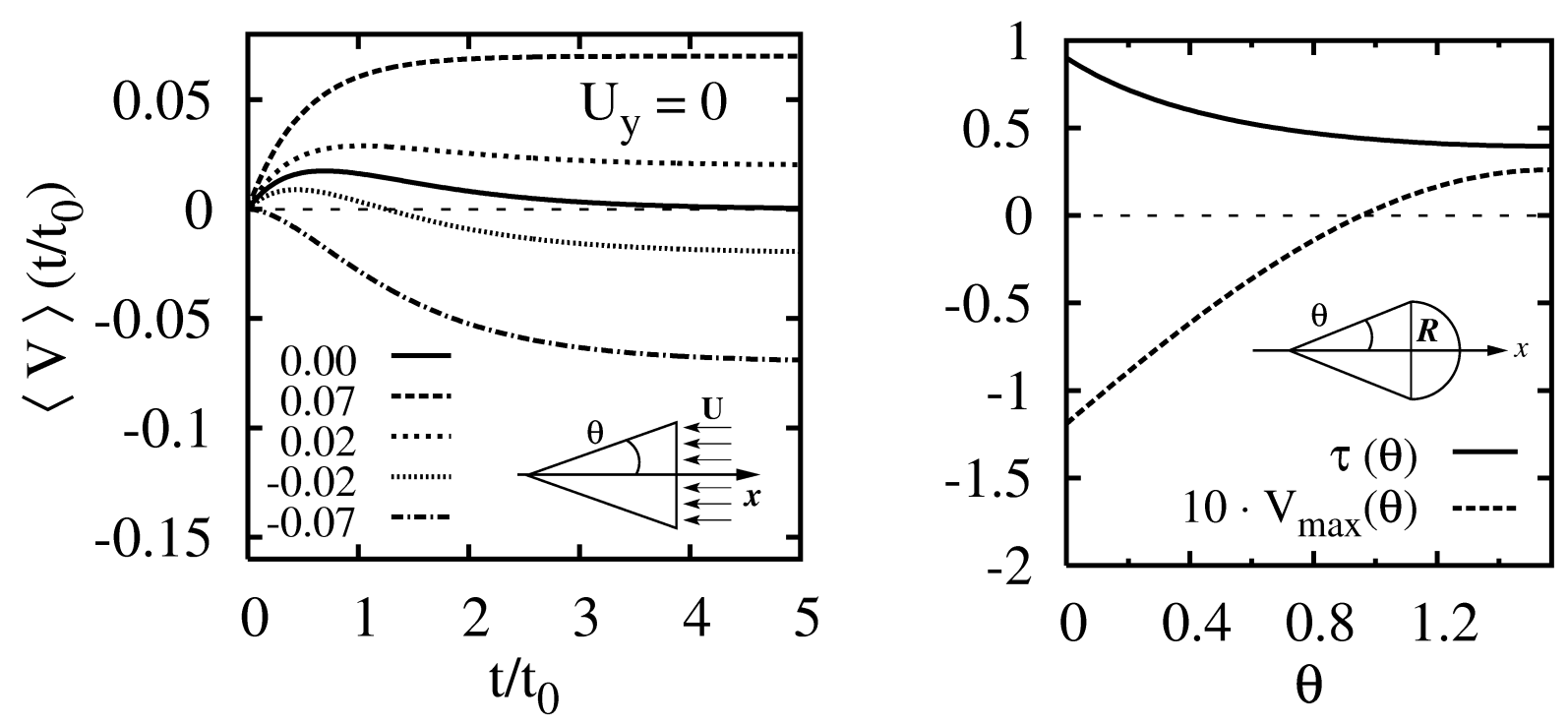

FIG. 4: (a) A triangular motor (apex angle $\theta=\pi / 6$, base length $L \sqrt{\rho}=1, \epsilon^{2}=m / M=0.05$ ) can swim even against a fluid drift velocity $\boldsymbol{U}=\sqrt{M /\left(k_{B} T\right)} \boldsymbol{u}$ during its relaxation in thermal equilibrium. (b) The direction of motion can even be inverted solely by changing the shape of the motor. The maximum velocity $V_{\max }$ and the relaxation time $\tau$ of a 'capped-triangular' motor (sketched in the inset) can be regulated by the opening angle $\theta$ which determines the Minkowski functionals in Eqs. (3) and (4).

area of its cylindrical cross section equals the pressure of the fluid on this side. Since the fluids on both sides are equilibrated at the same temperature there is no net force on the piston. The same is true if the piston can move freely and is in thermal equilibrium with the fluids on both sides. However, a piston with finite mass $M$ and not in equilibrium experiences kinematic effects due to momentum conservation when momentum is transfered by collisions, so that the net force depends on the shape of the piston.

The motor is also able to move against small fluid drifts. Repeating the analytic calculations with $\boldsymbol{u} \neq 0$ and confining the drift to the $x$-direction we find the mean velocity shown in Fig. 4(a) for a triangular motor with its apex pointing in negative $x$-direction. In the long time limit the motor equilibrates always to the drift velocity independent of sign and strength of the drift. For small drifts in the preferred direction - here the positive $x$-direction - the motor accelerates to a maximum average velocity $V_{\max }$ above the equilibrium value before it relaxes to the drift velocity $\boldsymbol{u}$. When applying a small drift in opposite direction, it can be seen that the motor starts to move in positive direction but changes its direction of motion after a certain time. When stopping it repeatedly before the direction is changed, an average motion against a fluid drift is achieved. 


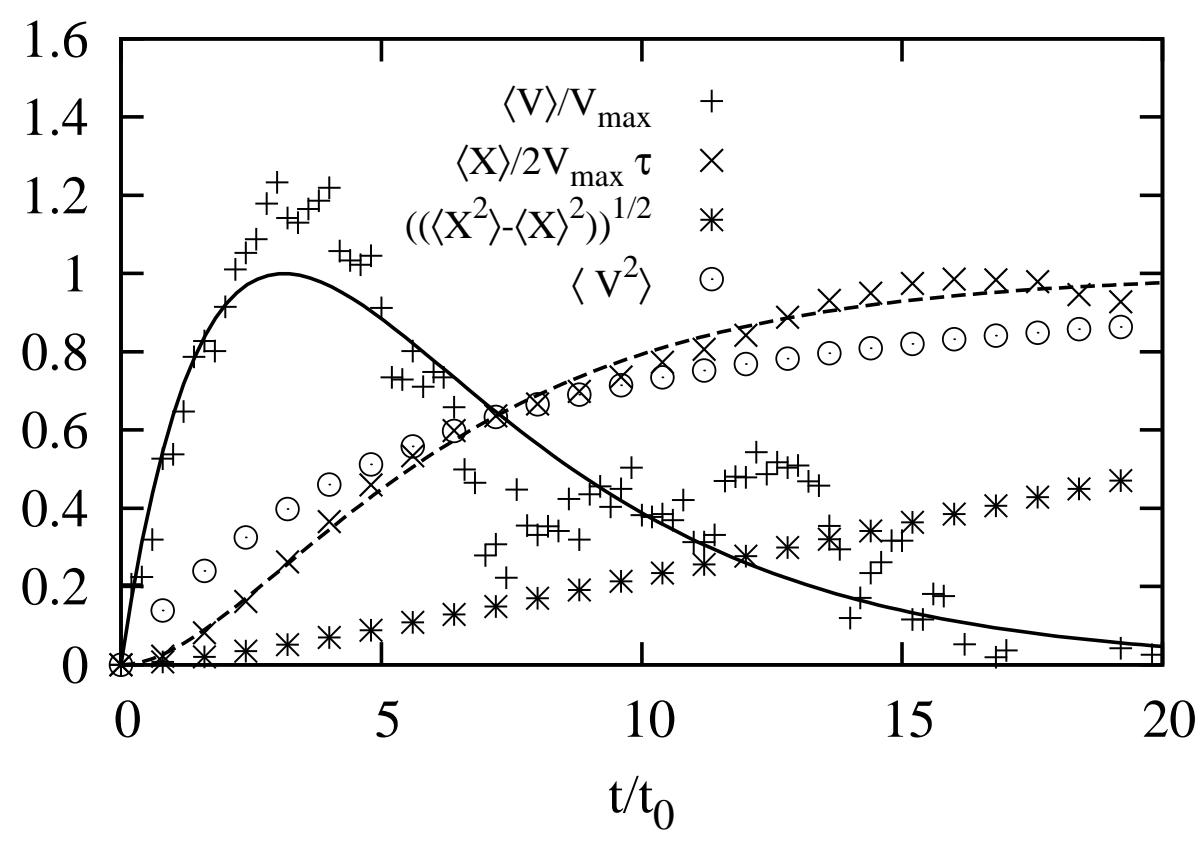

FIG. 5: Relaxation of a motor to thermal equilibrium: the molecular dynamics simulations of the LJ-fluid (symbols; averages over 400000 runs) can be well described by the analytic results in the ideal gas limit (lines) if the data are normalized by the maximum velocity $V_{\max }$ and the relaxation time.

Fig. 5 compares our analytic results with the numerical data from the molecular dynamics simulations. Evidently, a real fluid with Lennard-Jones interactions cannot be in quantitative agreement with calculations based on an ideal gas. Nevertheless, we find the same functional form for the non-equilibrium relaxation process given by the solution of Eq. (5), which confirms our theoretical understanding of this rectification mechanism. We showed that thermal fluctuations in a single heatbath can be used to get directed motion if (i) the motor is sustained in a non-equilibrium state by stopping it at periodically spaced sites on a one-dimensional track (or alternatively at periodic time intervals); (ii) the spatial symmetry is broken by an asymmetric shape of the motor which makes the non-stationary relaxation process also asymmetric. The shape-dependence of the nonequilibrium relaxation is used to get directed motion even against small fluid drifts. By changing the shape of the motor, it is possible to change the direction of motion and to control the average velocity of the motor.

[1] A. Einstein, Annalen der Physik 17, 549 (1905). 
[2] M. v. Smoluchowski, Annalen der Physik 21, 756-780 (1906).

[3] P. Reimann, Phys. Rep. 361, 57 (2002).

[4] R. Feynman, R. Leighton and M. Sands, The Feynman-Lectures on Physics I, (Addison-Wesley, Reading, 1963), Chap. 46.

[5] C. Van den Broeck, R. Kawai and P. Meurs, Phys. Rev. Lett. 93, 090601 (2004); New Journal of Physics 7, 10 (2005).

[6] J. Howard, The movement of kinesin along microtubules, Annual Review of Physiology 58, 703 (1996).

[7] B. Cleuren et al., Europhys. Lett. 77, 50003 (2007).

[8] G. Costantini et al., Phys. Rev. E 75, 061124 (2007).

[9] S. Sporer, Ein Modell für gerichtete Brownsche Bewegung in einem einzelnen Wärmebad, diploma thesis (Erlangen, 2006).

[10] K. Mecke and D. Stoyan, Morphology of Condensed Matter, Lecture Notes in Physics, Vol. 600 (Springer, Berlin, 2002). 